\title{
Transient mitochondrial DNA double strand breaks in mice cause accelerated aging phenotypes in a ROS-dependent but p53/p21-independent manner
}

\author{
Milena Pinto ${ }^{1,8}$, Alicia M Pickrell ${ }^{2,8,9}$, Xiao Wang ${ }^{3,8,10}$, Sandra R Bacman ${ }^{1}$, Aixin Yu ${ }^{4}$, Aline Hida ${ }^{1}$, Lloye M Dillon ${ }^{5,11}$, Paul D Morton ${ }^{6,7}$, \\ Thomas R Malek ${ }^{4}$, Siôn L Williams ${ }^{1}$ and Carlos T Moraes ${ }^{*, 1,2,3,5}$
}

\begin{abstract}
We observed that the transient induction of mtDNA double strand breaks (DSBs) in cultured cells led to activation of cell cycle arrest proteins (p21/p53 pathway) and decreased cell growth, mediated through reactive oxygen species (ROS). To investigate this process in vivo we developed a mouse model where we could transiently induce mtDNA DSBs ubiquitously. This transient mtDNA damage in mice caused an accelerated aging phenotype, preferentially affecting proliferating tissues. One of the earliest phenotypes was accelerated thymus shrinkage by apoptosis and differentiation into adipose tissue, mimicking age-related thymic involution. This phenotype was accompanied by increased ROS and activation of cell cycle arrest proteins. Treatment with antioxidants improved the phenotype but the knocking out of p21 or p53 did not. Our results demonstrate that transient mtDNA DSBs can accelerate aging of certain tissues by increasing ROS. Surprisingly, this mtDNA DSB-associated senescence phenotype does not require $\mathrm{p} 21 / \mathrm{p} 53$, even if this pathway is activated in the process.
\end{abstract}

Cell Death and Differentiation (2017) 24, 288-299; doi:10.1038/cdd.2016.123; published online 2 December 2016

Aging is a highly complex, yet poorly understood, orchestration of cell signaling events resulting in metabolic and regenerative declines that lead to cell death, cell cycle arrest, senescence, or terminal differentiation. ${ }^{1}$ Nuclear DNA damage is considered a primary causal factor in aging. ${ }^{2}$ Premature aging phenotypes have been widely observed in mouse models lacking nDNA repair enzymes. ${ }^{3-7}$ p53 is one of the most extensively studied proteins in modern biology, playing a central role in responding to diverse types of nDNA damage by coordinating cell fate, often in the context of either promoting aging or suppressing cancerous processes. ${ }^{8}$ Genes that are transcriptionally activated by p53 have been implicated in multiple models of aging. ${ }^{9-11}$

Mitochondria are tied to the aging process, through their involvement in apoptosis, energy production or the generation of signaling molecules such as reactive oxygen species (ROS). ${ }^{12-15}$ Mitochondria have multiple copies of their own genome, which encodes subunits for the different complexes of the oxidative phosphorylation (OXPHOS) system. ${ }^{16}$ It is suggested that decline in mitochondrial function caused by
mtDNA damage contributes to cellular aging. ${ }^{17,18}$ However, often times in aged tissues, mtDNA mutational levels do not exceed the threshold sufficient to cause mitochondrial dysfunction. ${ }^{19,20}$ Moreover, low levels of ROS have been shown to signal extension of life span in different organisms. ${ }^{21,22}$ Presently, it is unclear how and to what extent mtDNA damage contributes to cellular senescence or aging phenotypes. In the present study, we used mitochondriatargeted restriction endonucleases to induce mtDNA damage in different systems. After observing decreased cell growth in vitro and a progeroid-like phenotype in vivo, we analyzed potential molecular mediators of these phenotypes.

\section{Results}

MtDNA double-stranded breaks cause defects in cellular growth and is associated with an upregulation of nuclear cell cycle checkpoint signaling. To determine how mtDNA damage contributes to cell growth, we utilized a

${ }^{1}$ Department of Neurology, University of Miami Miller School of Medicine, Miami, FL 33136, USA; ${ }^{2}$ Neuroscience Graduate Program, University of Miami Miller School of Medicine, Miami, FL 33136, USA; ${ }^{3}$ Graduate Program in Cancer Biology, University of Miami Miller School of Medicine, Miami, FL 33136, USA; ${ }^{4}$ Department of Microbiology and Immunology, University of Miami Miller School of Medicine, Miami, FL 33136, USA; ${ }^{5}$ Department of Cell Biology and Anatomy, University of Miami Miller School of Medicine, Miami, FL 33136, USA; ${ }^{6}$ Department of Neurosurgery, University of Miami Miller School of Medicine, Miami, FL 33136 , USA and ${ }^{7}$ Center for Neuroscience Research, Children's National Medical Center, Washington, DC 20010, USA

*Corresponding author: CT Moraes, Department of Neurology, University of Miami Miller School of Medicine, 1420 NW 9th Avenue, Rm.229, Miami, FL 33136, USA. Tel: 305243 5858; Fax: 305243 6955; E-mail: cmoraes@med.miami.edu

${ }^{8}$ These authors contributed equally to this work.

${ }^{9}$ Present address: Surgical Neurology Branch, National Institute of Neurological Disorders and Stroke, National Institutes of Health, Bethesda, MD 20892, USA.

${ }^{10}$ Present address: Department of Molecular and Cellular Oncology, University of Texas MD Anderson Cancer Center, Houston, TX 77030, USA.

${ }^{11}$ Present address: OncoTAb, Charlotte, NC 28223, USA.

Abbreviations: mtDNA, mitochondrial DNA; DSBs, double strand breaks; ROS, reactive oxygen species; OXPHOS, oxidative phosphorylation; COX8, cytochrome $c$ oxidase subunit VIII; Cdkn1a, cyclin-dependent kinase Inhibitor 1 A (p21/Cip1); MDM2, Mouse double minute 2; NAC, N-acetyl-cysteine; TUNEL, terminal deoxynucleotidyl transferase dUTP nick end labeling; DOX, doxycycline; DEXA, dual-energy X-ray absorptiometry; DN, double negative, CD4 - /CD8 - ; DP, double positive CD4+/CD8+; PGC, peroxisome proliferator activated receptor gamma coactivators; PPAR $\gamma$, peroxisome proliferator activated receptor gamma; ADRP, adipose differentiation related protein; Systemic ${ }^{\text {Ind }}$ mito-Pstl, systemic-inducible mitochondrial-targeted Pstl

Received 19.7.16; revised 16.9.16; accepted 26.9.16; Edited by N Chandel; published online 02.12.16 
a

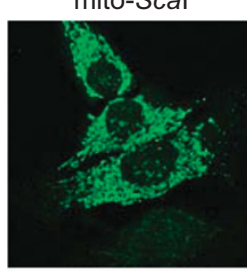

d

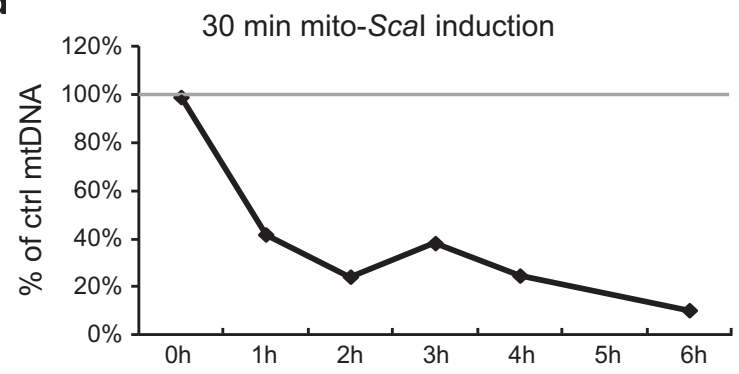

f
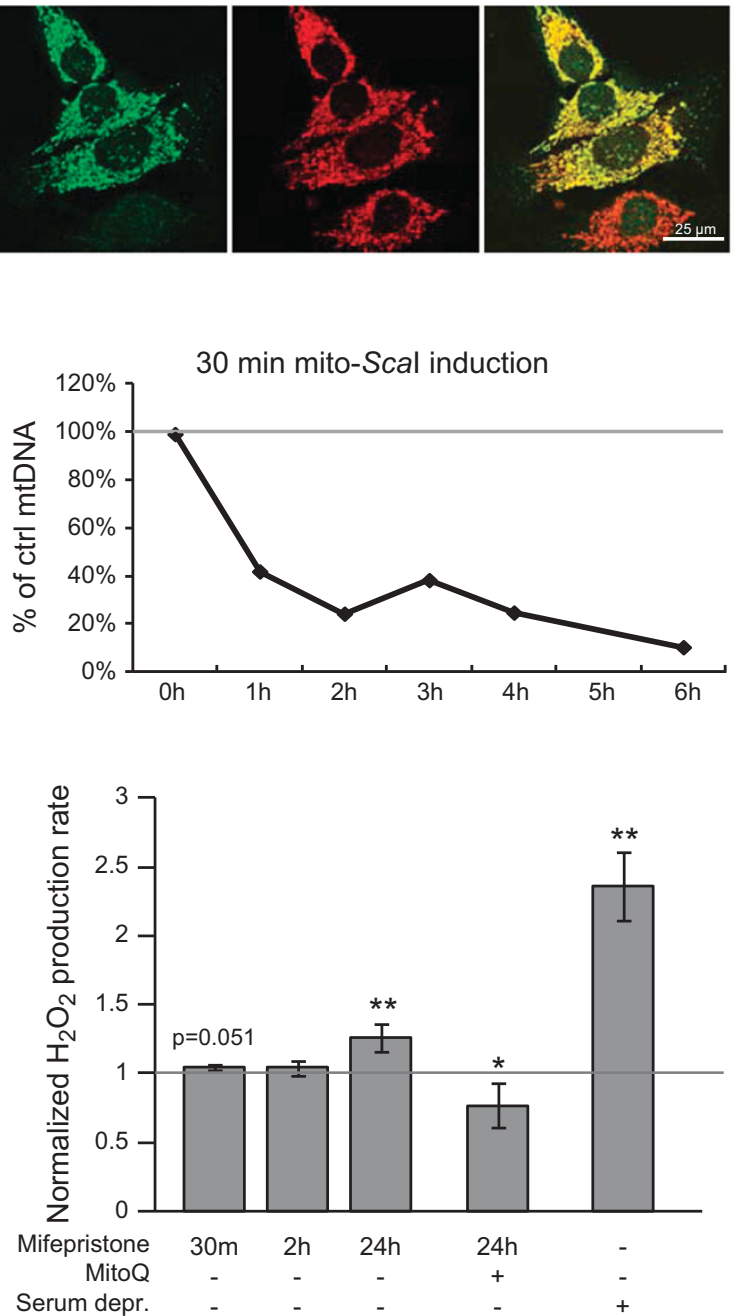

b
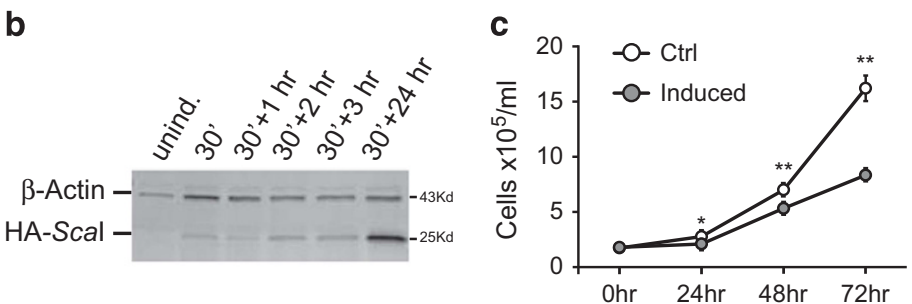

e $\quad \square$ Uninduced

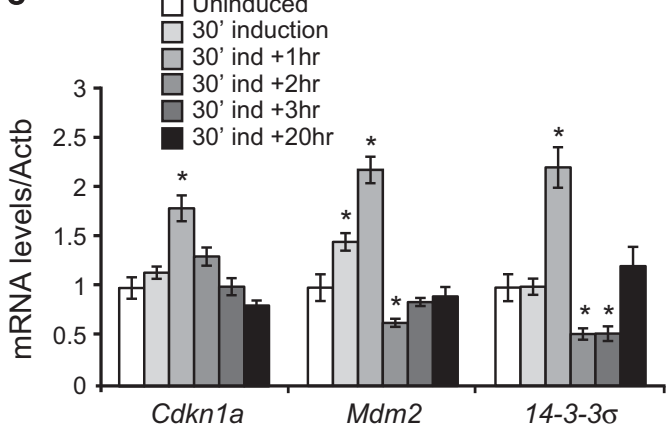

g

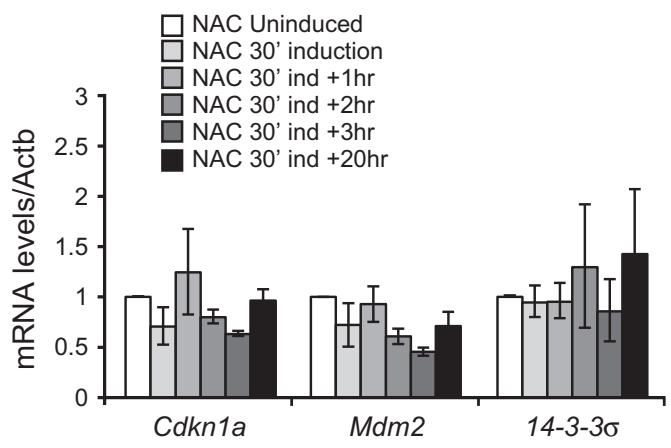

Figure 1 MtDNA DSBs lead to a ROS-dependent activation of p53 pathway in cultured cells. (a) Immunocytochemistry of mito-Scal cells using antibody against HA (green) and MitoTracker Red (red) after $10 \mathrm{nM}$ mifepristone induction for $2 \mathrm{~h}$. Images were captured at $\times 20$. (b) Representative western blotting showing mito-Scal (with HA tag) is induced by administrating $10 \mathrm{nM}$ mifepristone for different lengths of time. (c) Quantification of Southern blot for mtDNA and nuclear rDNA levels in mito-Scal cells at different time points after $10 \mathrm{nM}$ mifepristone induction for 30 min showing mito-Scal depletes mtDNA. (d) Growth curve over 3 days after 30 min mifepristone induction of mito-Scal cells and non-induced controls $(n=3)$. (e, g) Real-time RT-PCR of 14-3-3 $\sigma$, Mdm2, and p21 (Cdkn1a) in mito-Scal cells after 30 min of mifepristone induction and 1-3, and $20 \mathrm{~h}$ recovery without $(\mathbf{e})$ or with $(\mathbf{g}) 24 \mathrm{~h}$ pre-treatment with NAC $(n=3)$. (f) Rate of hydrogen peroxide production detected by Amplex Red assay in mito-Scal cells. Serum deprivation for $24 \mathrm{~h}$ was used as a positive control, and Mito Q was used as a negative control. Values are presented as mean \pm STDEV. ${ }^{*} P<0.05,{ }^{\star \star} P<0.01,{ }^{* \star *} P<0.001$

hepatocyte-derived cell line stably expressing a mifepristoneinducible mitochondrial-targeted endonuclease mito-Scal ${ }^{23}$ (Figure 3b). Mito-Scal co-localized with the mitochondrial marker MitoTracker Red red (Figure 1a) and was detected shortly after the addition of mifepristone, with a peak at 24-48 $\mathrm{h}$ of induction (Figure 1b). Its expression after 30-min induction led to a rapid reduction of mtDNA levels (Figure 1d) and to a decrease in cell proliferation in the following days (Figure 1c).

After mtDNA double strand breaks (DSBs) we observed an upregulation of the cell cycle arrest protein, p21 (Cdkn1a) transcript (Figure 1e). We also observed an increase in transcript levels of two other genes activated by p53: Mdm2, negative regulator of $\mathrm{p} 53$, and 14-3-3 $\sigma$, a cell cycle checkpoint controller (Figure 1e). These transcriptional responses decreased $2 \mathrm{~h}$ after the mito-Scal stimulus (Figure 1e). These data suggested that acute mtDNA damage triggered the activation of nuclear signaling pathways that control the progression of cell cycle, possibly prior to mitochondrial dysfunction: COX I protein level (Supplementary Figures S1A-B), and cytochrome $c$ oxidase enzymatic activity (ref. 23) decreased $24 \mathrm{~h}$ after the induction. P38 and JNK, which also participate in a signaling cascade controlling cellular responses to stress, were not altered ${ }^{24,25}$ (Supplementary Figures S1A, C).

Antioxidants abolish the transcriptional response present after mtDNA damage. To determine the mechanism regarding how the mtDNA damage triggered a p53/p21 
response, we investigated whether ROS was involved in the signaling in the mito-Scal inducible cell line. We measured hydrogen peroxide production after mtDNA damage, using serum deprivation as a positive control, ${ }^{26}$ and we detected a small increase in $\mathrm{H}_{2} \mathrm{O}_{2}$ after 30 min of mito-Scal induction, but it fell short of significance $(p=0.051)$. We did observe a larger increase in $\mathrm{H}_{2} \mathrm{O}_{2}$ after $24 \mathrm{~h}$ whereas in the presence of MitoQ, a mitochondrial-targeted antioxidant, there was a significant decline in ROS production after mtDNA damage (Figure 1f). We also tested whether antioxidant treatment could abolish the mtDNA damage signaling. Indeed, $\mathrm{N}$-acetyl-cysteine (NAC) treatment neutralized changes in cell cycle arrest gene expression in cells with mito-Scal induction (Figures 1e and $\mathrm{g}$ ). These data suggested that increased ROS mediates the signal between mtDNA DSBs, p53 signaling and cell growth changes.

The upregulation in nuclear transcriptional response is not due to nuclear DNA damage. To ensure that the cell growth inhibitory effects observed were not due to leakage of mito-Scal into the nucleus, we performed TUNEL and $\gamma$-H2AX assays. Mito-Scal cells were either induced with mifepristone or treated with staurosporine, an inducer of nDNA fragmentation. We observed TUNEL (Figure 2a) and $\gamma$-H2AX (Figure 2b) positive nuclei in cells treated with staurosporine but not in cells expressing mito-Scal for $24 \mathrm{~h}$.

To further ensure that our restriction endonucleases were specifically targeted to mitochondria, we added the same COX8 mitochondrial-targeting sequence to a different endonuclease $(P s t)$ and expressed it in $\rho^{\circ}$ and $\rho^{+}$cells. Mito- $P s t 1$ promoted significant increase in phosphorylated MDM2 and p21 protein levels in $\rho^{+}$cells but not in $\rho^{\circ}$ cells (Figures $2 c-e$ ). Etoposide (nDNA damaging agent) was used as positive control and induced an increase in phosphorylated MDM2 and p21 levels in both $\rho^{\circ}$ and $\rho^{+}$cells (Figure 2c-e).

These results support that the decline in proliferation and the upregulation in nuclear transcriptional response controlling cell cycle were due to damage to mtDNA and not to an unspecific cleavage of nDNA.

Systemic and inducible expression of mito-Pst in a transgenic mouse model. To investigate the response to mtDNA DSBs in vivo, we developed a mouse model with inducible mito-Pstl expression. This mouse model ubiquitously expresses two transgenes: a tetracycline-inducible, mitochondrial-targeted Pstl endonuclease (mito-Pstl) and a reverse tetracycline transactivator protein (rtTA) under the control of the ubiquitous Rosa26 promoter $^{27}$ (Figure 3a). We will refer to this double-transgenic as 'Systemic ${ }^{\text {Ind }}$ mito-Pst' mouse. To avoid developmental defects that may occur with early mito-Pstl exposure, mito- $P s t l$ expression was induced at 3 months of age for only 5 days (Figure $3 d$ ). DOX food was given to both Systemic ${ }^{\text {Ind }}$ mito-Pstl and control mice.

Mito-Pstl expression was relatively low in brain, heart and skeletal muscle, whereas levels were higher in liver, thymus, lung and kidney (Figure 3c). Mito-Pstl expression was transient and detectable by western blot only during the first week after the 5-day induction period even in tissues with the highest expression (Figure 3d). a
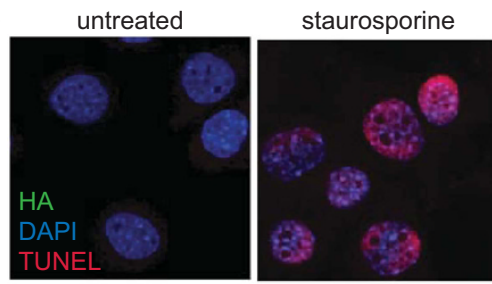

mito-Scal induction
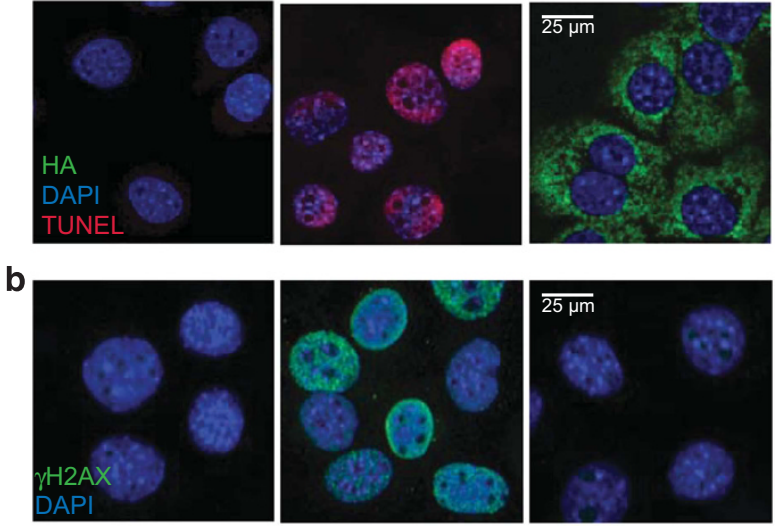

C
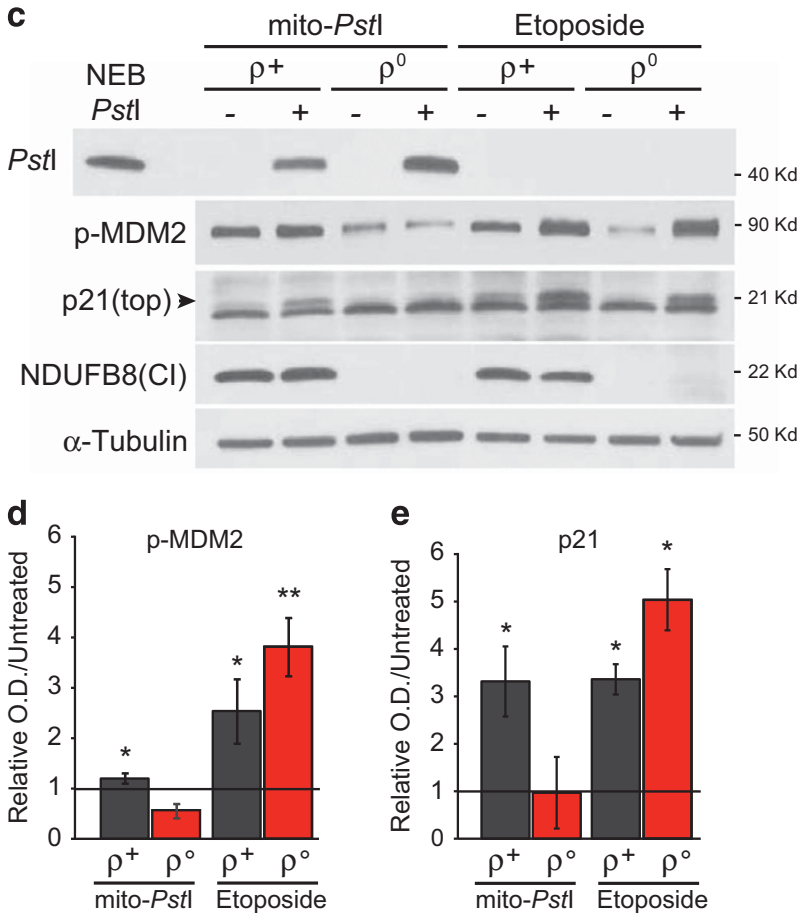

Figure 2 MtDNA DSBs trigger a p53-dependent response without nuclear DNA damage. (a) Representative TUNEL staining detecting DNA fragmentation (red) after inducing apoptosis with staurosporine or mito-Scal expression for $24 \mathrm{~h}$. Cells were counterstained with DAPI (blue) to show the nucleus and anti-HA (green) to show Scal expression $(n=3)$. (b) Representative $\gamma \mathrm{H} 2 \mathrm{AX}$ staining (green) after staurosporine or mito-Scal expression for $24 \mathrm{~h}$. Cells were counterstained with DAPI (blue) $(n=3)$. (c) Western blotting of phosphorylated MDM2, p21 in gorilla $\rho^{+}$ and $\rho^{\circ}$ cells either treated with $10 \mu \mathrm{M}$ Etoposide (ETP, DMSO vehicle) or transfected with mito-Pstl (mito-GFP as ctrl) for $24 \mathrm{~h}$. (d-e) Quantification of phospho-MDM2 (d) and p21 (e) protein levels after ETP treatment or mito-Pstl transfection normalized to $\beta$-actin, in relation to the not-stressed cells $(n=3)$. The marked line indicates the control level in untreated cells. Values are presented as mean \pm STDEV. ${ }^{*} P<0.05$, ${ }^{\star \star} P<0.01,{ }^{\star \star \star} P<0.001$

To test whether mito-Pstl was functional and whether it cleaved mtDNA in vivo, we measured mtDNA levels. MtDNA was reduced in the lung and liver, immediately following a 5-day induction (Figure 3e), but less so after 3 months (Figure 3f). Kidney and muscle showed a mild, not significant 
mtDNA depletion after 5 days of induction, but curiously, there was depletion after 3 months. MtDNA depletion was not observed in the brain, thymus, heart and testis. Previous studies from our laboratory have shown that mtDNA DSBs lead mostly to mtDNA depletion, but also to the formation of mtDNA deletions in post mitotic tissues, albeit at low levels. ${ }^{28}$

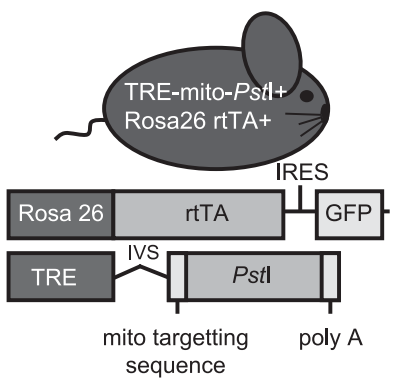

C

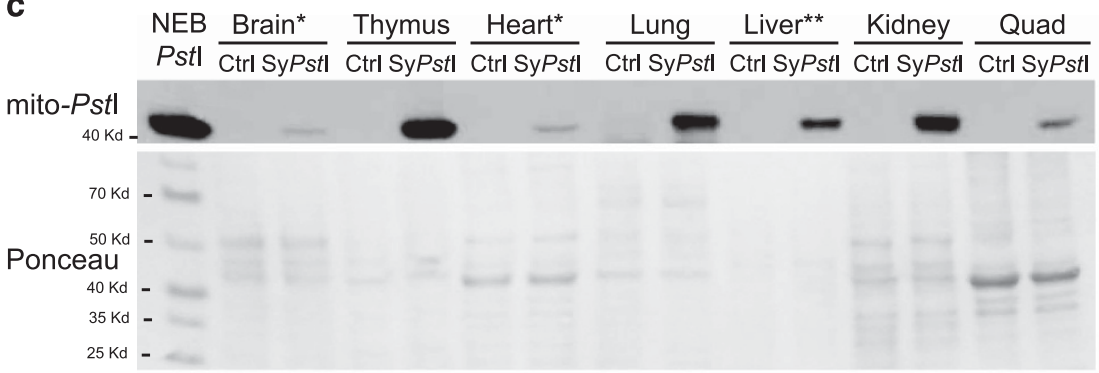

d

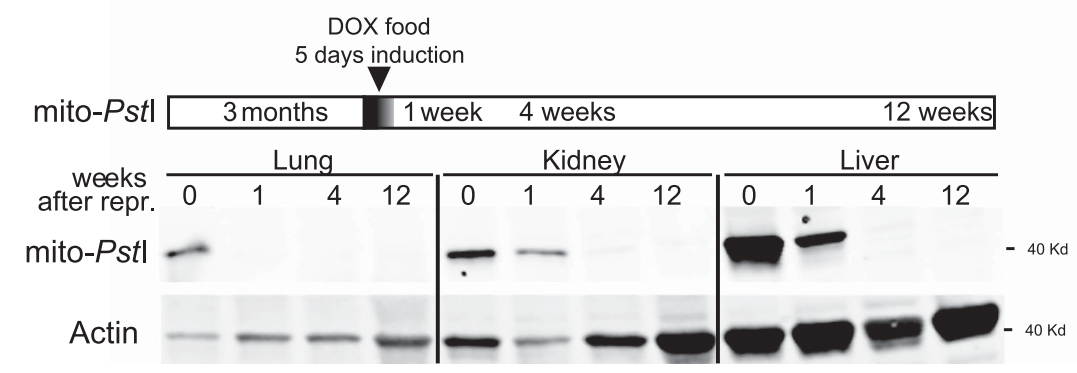

e

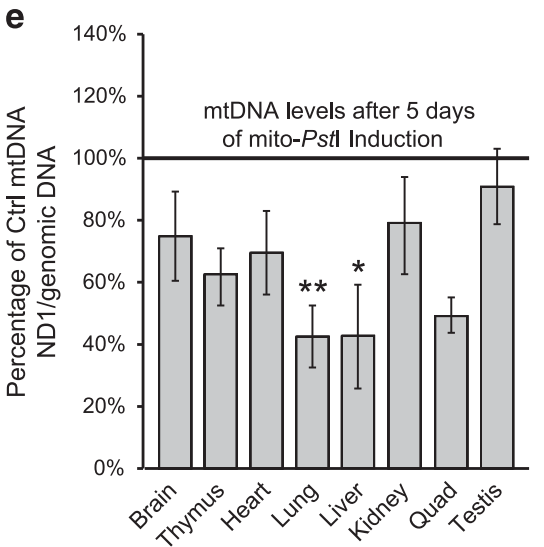

Figure 3 Characterization of transient and systemic expression of mito-Pstl in transgenic mice. (a) Schematic representation of the Systemic ${ }^{\text {Ind }}$ mito- $P s t t$ mouse and the transgenic constructs it harbors. (b) mtDNA map showing the targeted sites of Pstl (scissors) and Scal (stars). Black arrows denote protein-coding genes. (c) Western blotting using anti-Pstl antibody to detect mito-Pstl expression in Systemic ${ }^{\text {Ind }}$ mito-Pstl (SyPstl) mice tissues at 3 months of age after a 5-day induction (brain, thymus, heart, lung, liver, kidney, quadriceps). NEB (New England Biolabs, Ipswich, MA, USA) Pstt restriction enzyme was used as a positive control. (d) Cartoon of mito-Pstl induction paradigm (5-days induction at 3 months of age). The lower part of the panel shows a western blot for Pstt in Systemic ${ }^{\text {Ind }}$ mito-Pstl animal tissues immediately, 1 week, 4 weeks, and 12 weeks after induction in lung, kidney and liver, tissues with the highest mito-Pstl expression. (e) Quantitative-PCR quantification of mtDNA/nuclear DNA ratios of tissues from the Systemic ${ }^{\text {Ind }}$ mito-Pstl mice, and age-matched controls immediately after the 5-days induction (left pane) or after 3 months, when animals were 6 months old (right panel) ( $n=4-5$ / group). All mice in these analyses were males. Values are presented as mean \pm S.E.M. $\left({ }^{\star} P<0.05,{ }^{* \star} P<0.01,{ }^{\star \star \star}{ }^{*} P<0.001\right)$ 
Although we were able to detect the presence of two types of mtDNA deletions in heart and brain (Supplementary Figure S2A), the levels were low $(<1 \%)$ and therefore unlikely to have functional consequences.

Systemic ${ }^{\text {Ind }}$ mito-Pst mice develop signs resembling premature aging. Systemic ${ }^{\text {Ind }}$ mito-Pstl mice appeared normal in the first 10-12 weeks following 5-day induction; however, 6 months after DOX exposure, the animals began to present phenotypes resembling aged mice (Figure 4k). One of the first noticeable phenotypes was kyphosis and fur graying (Figure 4a). The fur graying, which has been linked to an inability to maintain melanocyte stem cell pools, ${ }^{5}$ spread from ventral toward dorsal side. To test whether there was a defect in hair follicle regenerative cell pools in our model, we induced the anagen hair phase by depilating the mice. We observed that Systemic ${ }^{\text {Ind }}$ mito-Pstl mice had markedly delayed fur re-growth (Figure 4b). After a second round of depilation, we observed additional fur loss and graying (Figure 4c). Histologically, hair follicles were defective and less in number (Figure 4f).

Because hair follicles are highly mitotic, we also analyzed other mitotic tissues of the Systemic ${ }^{\text {Ind }}$ mito-Pstl mice to determine their vulnerability. Blood chemistry panels showed decreases in phosphorus and total proteins in the serum, an effect commonly associated with mal-absorption related to abnormalities of the small intestine (Figure 4d). In addition, we also found significant shrinkage of the testes at 6 months of age (Figures $4 \mathrm{e}$ and j). Dual-energy X-ray absorptiometry (DEXA) scans revealed decreases in bone mineral density, indicative of osteoporosis (Figure $4 \mathrm{~g}$ ), accompanied by significant decreases in total fat, percentage of body fat and lean tissue mass (Figure $4 \mathrm{~h}$ ). A decrease in fat was also evident in the dermal layers of the skin (Figure 4f). Moreover, Systemic ${ }^{\text {Ind }}$ mito-Pstl mice also had significant declines in white blood cell populations and signs of anemia (Figure 4i).

Brain, thymus, liver, quadriceps and testis weight, as well as total body weight, were significantly lower in 1-year-old Systemic ${ }^{\text {Ind }}$ mito-Pstl mice (Figure 4j). Mito-Pstl induction did not result in premature death in any of the females monitored (up to 2 years of age); however, males often died before 18 months of age.

Accelerated thymus involution in response to mitoPstl. Immediately after 5-day induction, Systemic ${ }^{\text {Ind }}$ mito-Pst mice had an acute and severe decrease in both thymic weight and thymocyte count (Figures $5 a$ and b). Small populations of lymphoid progenitor cells in the thymus differentiate and give rise to mature $\mathrm{T}_{\text {cells }}{ }^{29}$ (Figure $5 \mathrm{~g}$ ). We analyzed the total thymocyte population for maturity stages after the 5-day induction. Double positive cells (CD4 +/CD8+) (DP) were significantly decreased, whereas mature $T$ cells (CD4+/CD8- or CD4-/CD8+) were significantly increased in the Systemic ${ }^{\text {Ind }}$ mito-Pstl mice (Figures $5 \mathrm{c}$ and $d$ ). These findings suggest that mtDNA damage impairs thymocyte development.

Further examination of the DN thymocyte population revealed increase in CD44+/CD25 - (DN1) cell population (Figures $5 \mathrm{e}$ and $\mathrm{f}$ ); this marks the first stage in thymocyte development (Figure $5 \mathrm{~g}$ ). The accumulation of DN1 cells indicates delayed or impaired transition to DN2 (CD44+/CD25+) that may explain the decreased numbers of DP thymocytes in these mice. We also observed that, at 3 months, thymus underwent apoptosis (cleaved caspase-3 reactivity) (Figure $5 \mathrm{~h}$ ) and senescence (marked by non-acidic $\beta$-galactosidase activity) (Figure 5i), mostly in the thymic cortex, a region enriched for progenitor and developing cells (Figures 5h-i).

The thymus of Systemic ${ }^{\text {Ind }}$ mito-Pstl mice could degenerate due to an OXPHOS defect, related to mtDNA depletion. We detected a trend, even if not significant, towards mtDNA depletion (Figure 3e) and COXI protein level decreased after 5 days of induction (Supplementary Figures S1D, E). Paradoxically, we observed a significant, 7-8-fold increase in mitochondrial encoded COXI and ND1 mRNA levels in these mice (Figure $5 \mathrm{j}$ ). Transcript levels of PGC-1 $a$ and PGC-1 $\beta$, the master regulators of mitochondrial biogenesis, were also increased by $6-8$ fold in this group (Figures $5 j-k$ ). Normal agerelated thymic involution is associated with differentiation of thymocytes into adipose tissue, with adipocytes having increased mitochondrial markers. ${ }^{30}$ Therefore, we analyzed the expression of PPARy, a factor involved in pre-adipose tissue differentiation, ${ }^{31-33}$ and found $a \sim 17$-fold increase in its mRNA levels (Figure 5k). Accordingly, ADRP expression level (another marker of early adipocyte differentiation) was also elevated (Figure 5k). To determine whether these transcriptional regulations had a functional consequence in thymic cellular composition, we performed Oil Red $O$ staining to detect triglycerides and lipids. We observed a dramatic increase in lipid deposits in Systemic ${ }^{\text {Ind }}$ mito-Pstl mice thymus

\footnotetext{
Figure 4 Systemic ${ }^{\text {Ind }}$ mito-Pstl mice show age-related phenotypes in mitotic tissues. (a) Representative images of 6-month-old mice show the ventral graying of the fur of Systemic ${ }^{\text {Ind }}$ mito-Pstl mice compared to age-matched controls. (b) Representative images of the $2 \times 2 \mathrm{~cm}^{2}$ patch depilated to induce anagen hair follicle cycle in controls and Systemic ${ }^{\text {Ind }}$ mito-Pstt mice ( $n=5 /$ group). (c) Representative images of the graying of the fur in Systemic ${ }^{\text {Ind }}$ mito-Pstl mice that sustained two rounds of depilation. (d) Basic serum chemistry panel on 6-month-old control and Systemic ${ }^{\text {Ind }}$ mito-Pstl female mice $\left(n=11\right.$ controls, $n=9$ Systemic ${ }^{\text {Ind }}$ mito-Pstl). Values are presented as mean \pm S.E.M. $\left({ }^{*} P<0.05\right)$. (e) Representative picture of the difference in size of testes from a control and a Systemic ${ }^{\text {Ind }}$ mito-Pstl male at 6 months of age. (f) Hematoxylin and eosin stained samples of skin from the depilated region after 10 days. Systemic ${ }^{\text {Ind }}$ mito-Pstl mice have abnormal dermal and epidermal layers with decreased fat content (white, unstained layer) and cycling hair

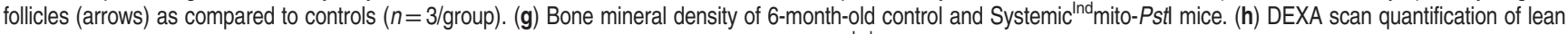
tissue, fat, and the total amount of tissue in the whole body of 6-month-old controls and Systemic Ind mito-Pstl males and females. ( $n=7-11 /$ group). (i) Blood panel on 6-month-old

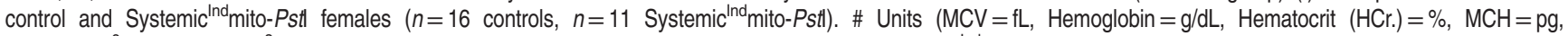
$\left.\mathrm{RBC}=\times 10^{6} / \mu l, \mathrm{WBC}=\times 10^{3} / \mu \mathrm{l}, \mathrm{MCHC}=\%\right)$. (j) Organ weight as percentage of the controls for Systemic ${ }^{\text {Ind }}$ mito-Pstt mice induced and killed immediately (3 months old, light red columns), after 3 months ( 6 months old, red columns) or after 6 months (12 months old, brown columns) ( $n=5-8 /$ group; males). Values are presented as mean \pm S.E.M. $\left({ }^{*} P<0.05,{ }^{* *} P<0.01,{ }^{* \star *} P<0.001\right)$. (k) Graphical representation for the induction schedule of Systemic ${ }^{\text {Ind }}$ mito-Pstl and color-code for the time points analyzed with relative phenotypes observed
} 
(Figure 5l). In conclusion, our data suggest that an accelerated aging-like response in the thymus was triggered by acute mtDNA damage, and resulted in a cascade of events that mimic natural mammalian thymic involution. ${ }^{30}$

MtDNA damage is associated with an upregulation of cell cycle arrest proteins in vivo. Mitotic tissues, such as hair follicles, thymus and reproductive organs, were preferentially affected (Figures 4 and 5) and these organs are known to have high $\mathrm{p} 53$ levels. ${ }^{34}$ Because mice harboring constitutively activated mutant $\mathrm{p} 53$ have progeroid phenotypes ${ }^{35,36}$ and we detected increased expression of p53 target genes in cultured cells after mtDNA DSBs (Figures 1 and 2), we tested whether p53 downstream markers were activated in the thymus of our Systemic ${ }^{\text {Ind }}$ mito-Pstl mice. Cdkn1a, Mdm2 and $14-3-3 \sigma$ transcripts and $\mathrm{p}-\mathrm{MDM} 2$ protein levels did not a

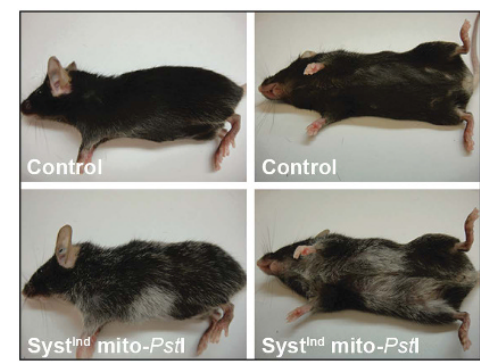

d

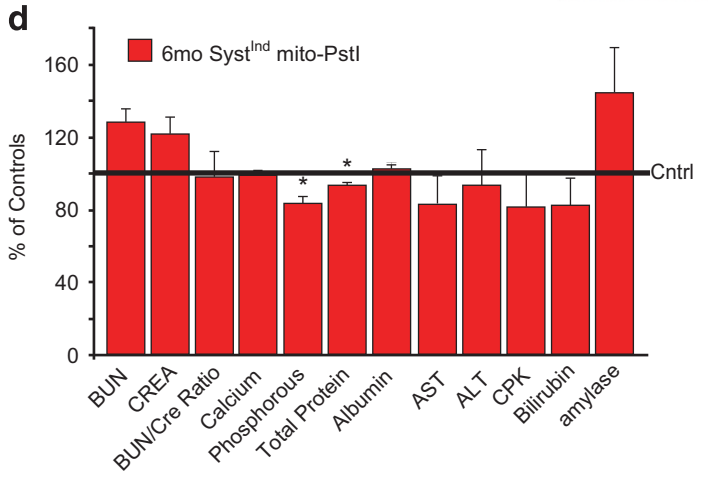

Day 0
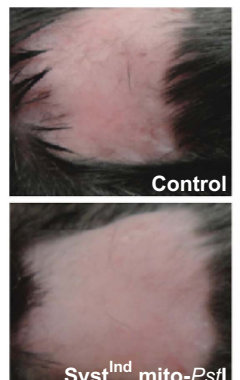

Syst ${ }^{\text {Ind }}$ mito-Pst
Day 10

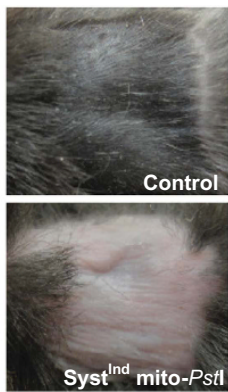

C

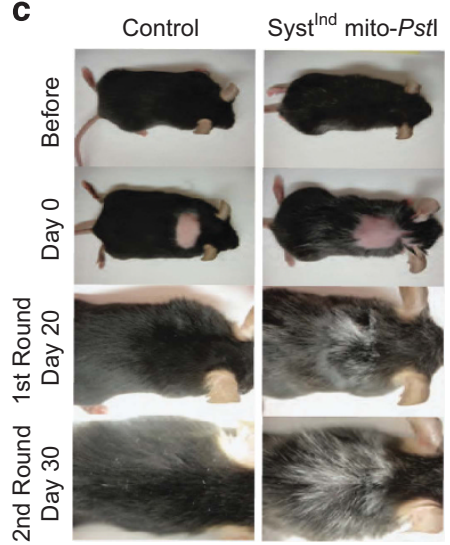

e

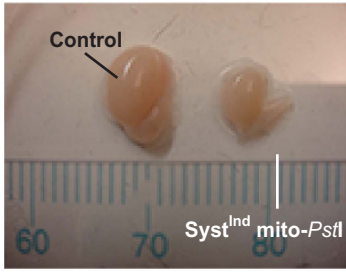

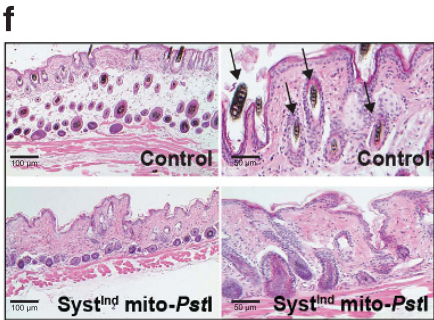
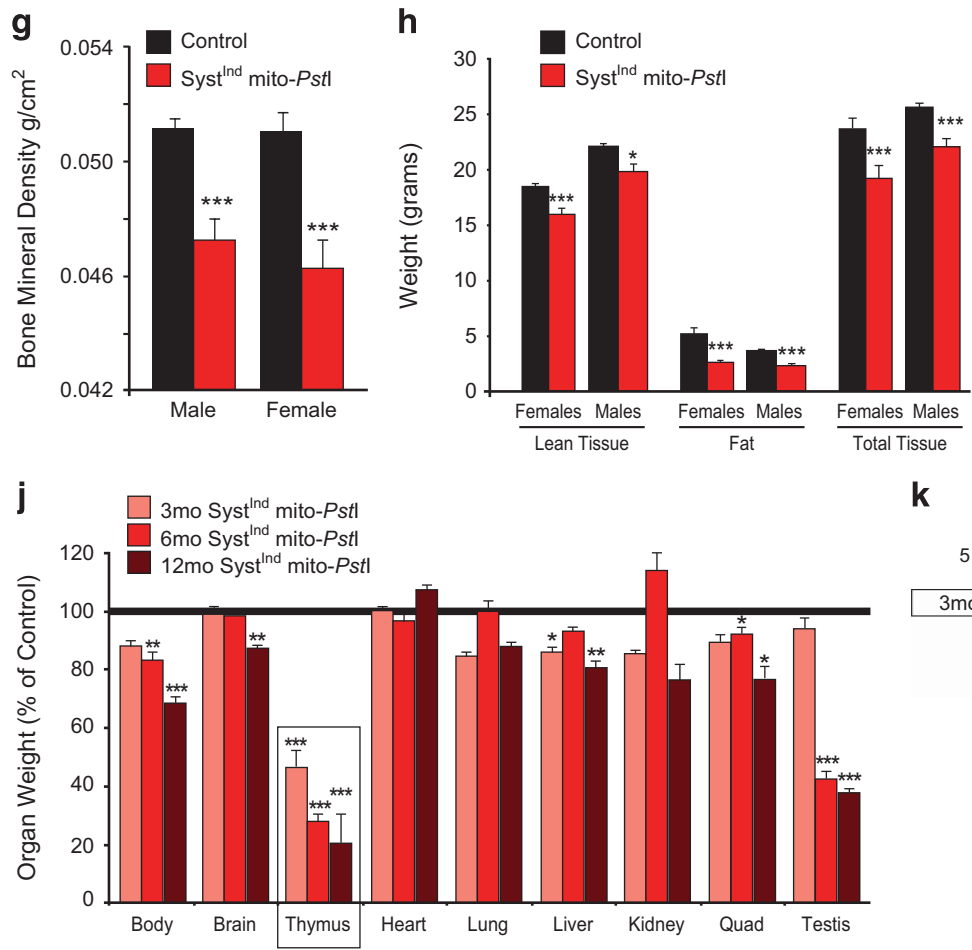

k

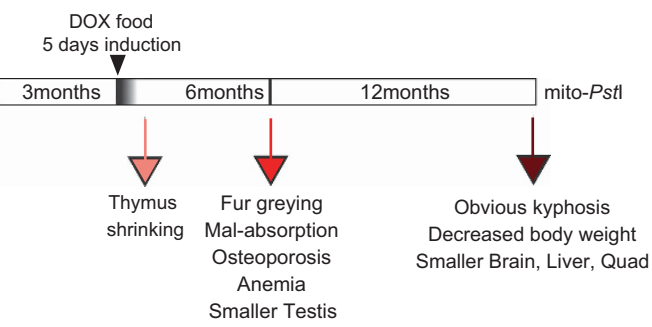


change after 5 days of mito-Pst induction (Figure 6a). However, since changes in the levels of these transcripts are commonly an early response to damage, we analyzed their levels at an earlier time point (2 days after induction). Cdkn1a and $14-3-3 \sigma$ transcripts levels were indeed upregulated (Figure 6c) and so was p-MDM2 (Figure 6d), indicating stabilization of $p 53 .^{37}$ These data indicated that cell cycle arrest signaling occurred before the accelerated thymic aging phenotype. We also analyzed p-p38/p38 and p-JNK1/JNK1 in thymus of 2 and 5 days induced mice but, similarly to the in vitro model, we did not detect activation of these pathways (Supplementary Figures S1D, E).

Using cultured cells, we showed that increased ROS mediated mtDNA DSB signaling pathways. Moreover, a previous report showed that NAC treatment ameliorated some of the aging phenotypes present in the Mutator mouse. ${ }^{38}$ To analyze if oxidative stress was involved in the aging-like phenotype in the thymus of Systemic ${ }^{\text {Ind }}$ mito-Pstl mice, we treated 3-month-old animals with NAC. This prevented the thymic involution caused by mtDNA DSBs (Figure 6e), suggesting the involvement of oxidative stress in this aginglike phenotype.

\section{p53/p21 pathway are not required for the mtDNA-induced progeroid phenotype. Our data showed that mtDNA DSBs} caused defects in cellular growth, which were associated with an upregulation of nuclear cell cycle checkpoint signaling in vitro (Figures $1 \mathrm{e}$ and $2 \mathrm{c}-\mathrm{e}$ ). We also showed how mtDNA DSBs cause a premature aging-like phenotype in vivo, particularly evident in proliferating cells, also associated with an activation of p53/p21 pathway in thymic homogenates. In order to determine whether the p53/p21 pathway is required for the progeroid phenotype observed in vivo, we knocked out p21 or p53 in the Systemic ${ }^{\text {Ind }}$ mito-Pstl mice by breeding the latter with $\mathrm{p} 21 \mathrm{KO}^{39}$ and $\mathrm{p} 53 \mathrm{KO}^{40}$ animals.

We induced $\mathrm{p} 21 \mathrm{KO} /$ Systemic $^{\text {Ind }}$ mito-Pstl and p53KO/Systemic $^{\text {Ind }}$ mito-Pstl mice and analyzed the involution of the thymus in 3-month-old animals. We still detected similar aginglike phenotypes (Figure 6f), indicating that p21 and p53 are not required for the acute phenotype caused by mtDNA damage.

To further assess whether $\mathrm{p} 21$ and $\mathrm{p} 53$ were required for the other progeroid-like phenotypes induced by mtDNA DSBs, we analyzed 6-month-old p21KO/ and p53KO/Systemic ${ }^{\text {Ind }}$ mitoPstl. These mouse lines develop multiple tumors at this age, particularly the p53KO animals. ${ }^{40}$ In our analysis we included only mice ( $\sim 40 \%$ of the total) that did not show evident signs of tumor formations. The graying of the fur observed in 6-monthold mito-Pstl was still present in the absence of p21 or p53 (Supplementary Figure S3A) as well as the delay in fur regrowth after depilation (Supplementary Figure S3B).

We measured the organ weight in 6-month-old animals and, apart from lung and liver that were smaller respectively in p21KO/Systemic ${ }^{\text {Ind }}$ mito-Pstl and p53KO/Systemic ${ }^{\text {Ind }}$ mito$P s t l$, we did not find any difference between the three groups of mice. The absence of p21 or of p53 did not alter these phenotypes (Supplementary Figure S3C), nor blood chemistry panels (Supplementary Figure S3D). We also analyzed the gross anatomy of intestine, testis and bone marrow by staining with $\mathrm{H} \& \mathrm{E}$ and detected no significant differences (Supplementary Figure S3E).

\section{Discussion}

Different studies show an accumulation of mtDNA mutations in aged tissues but, often, mtDNA mutational levels do not exceed the threshold sufficient to cause mitochondrial dysfunction. ${ }^{19,20}$ Presently, it is unclear how and to what extent mtDNA damage contributes to cellular senescence or aging phenotypes. Although large rearrangements of mtDNA are known to accumulate in aging tissues of mammals, the causative role of these mtDNA species in aging is still speculative.

In this study, we showed that a short induction of DSBs in mtDNA resulted in a premature aging-like phenotype in proliferating mouse tissues. MtDNA DSBs caused a p53 transcriptional response in vitro and, in some tissues, also in vivo but neither p53 nor p21 was required for the aging phenotype observed in vivo. We also showed that ROS play a role in the development of the phenotype and in the signaling process, as antioxidants blunted these responses.

MtDNA DSB damage response. Previous studies have suggested that there are shared nuclear proteins and responses after mtDNA damage: (1) Nuclear Ku proteins, involved in nuclear DSB repair, are associated with mtDNA DSB repair; ${ }^{41}$ (2) DNA Ligase III also has an alternatively spliced form that locates to the mitochondria with its function independent of its role in the base excision repair; ${ }^{42,43}$ (3) Besides its role in nuclear DSB repair, ATM kinase is involved in maintaining mitochondrial homeostasis; ${ }^{44,45}$ (4) Rad51 has been found to play critical roles in the maintenance of mitochondrial genome. ${ }^{46}$ The fact that several studies showed that DNA repair proteins are shared between the nuclear and mitochondrial compartments, suggests that damage to mtDNA, independent of nDNA damage, regulates classical signaling pathways as shown in this study. However, at this point, it is not clear if any of these nuclear DNA damage response sensors play a role in the response to mtDNA DSBs.

MtDNA DSBs affect cells with proliferative potential. MtDNA DSBs can signal the nucleus either by a sensor mechanism detecting the damage, or indirectly, by a defect in OXPHOS function. In our experience, mtDSBs lead to an mtDNA depletion as linearized molecules are rapidly degraded. ${ }^{47}$ However, other types of mtDNA mutations can also result from DSBs. Mito-Pstl expression was low in the brain and heart (Figure 3c), but when we measured mtDNA deletions in different tissues of 6-month-old animals, these tissues showed the highest levels of mtDNA deletions (Supplementary Figure S2B). We suspect that these deleted molecules were expanded due to the post-mitotic nature of brain and heart, since we detected no accumulation of mtDNA deletions in liver, the tissue with the highest mito-Pst expression (Supplementary Figure S2B). However, at 6 months, we observed no biochemical (assessed by measuring OXPHOS proteins levels in brain homogenates) or behavioral deficits (assessed by Morris water maze test) in the CNS (data not shown). We did observe a significant muscle wasting phenotype in Systemic ${ }^{\text {Ind }}$ mito-Pstl mice, which was attributed to the sensitivity and preferential loss 
a

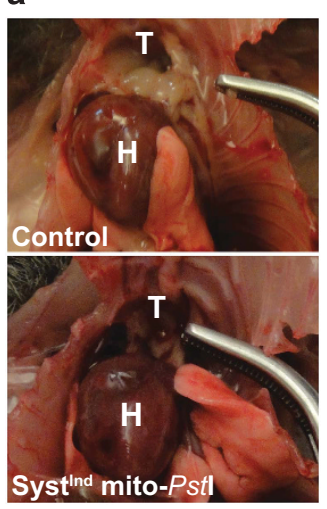

g

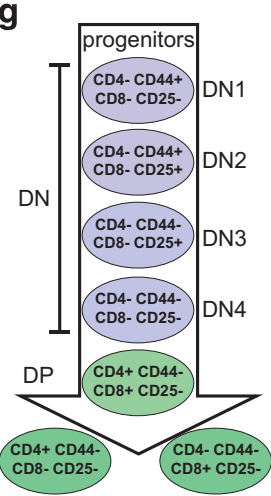

mature cells b

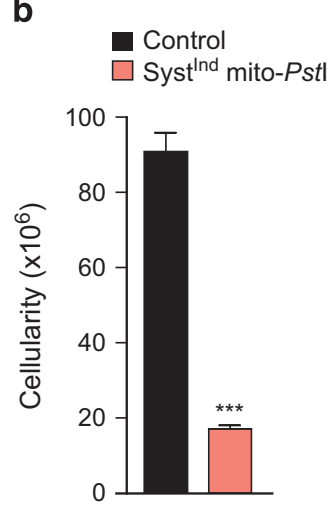

C

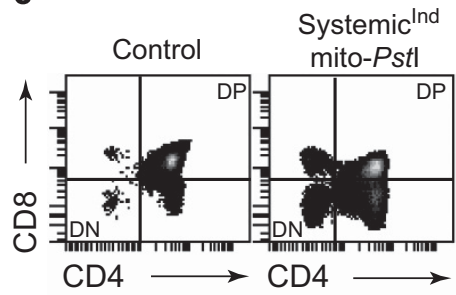

e

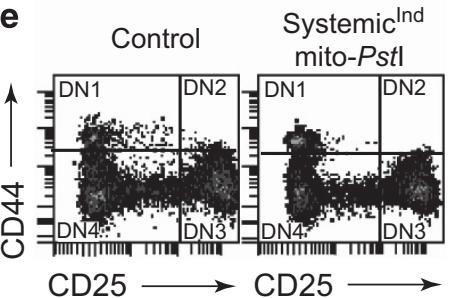

Gated on CD4-/CD8- cells
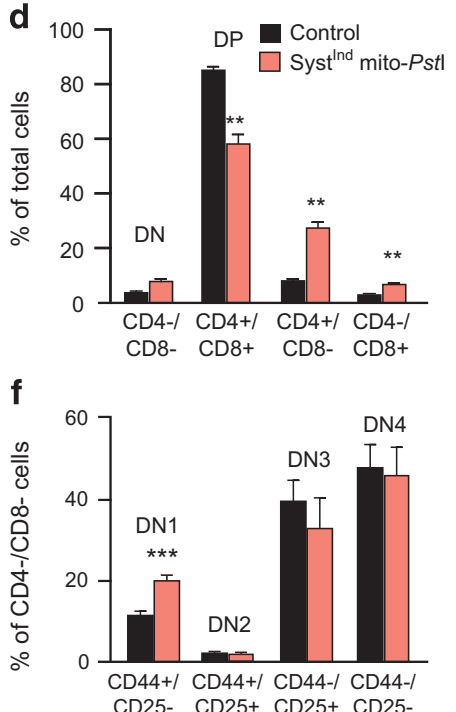

h

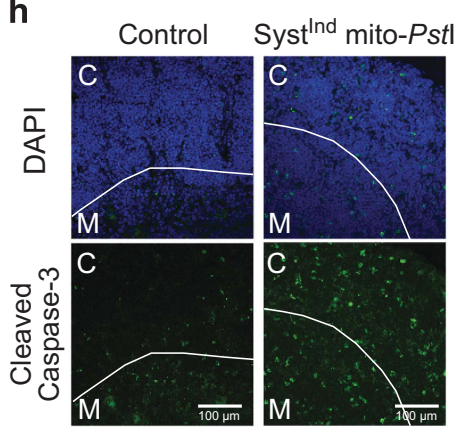

j
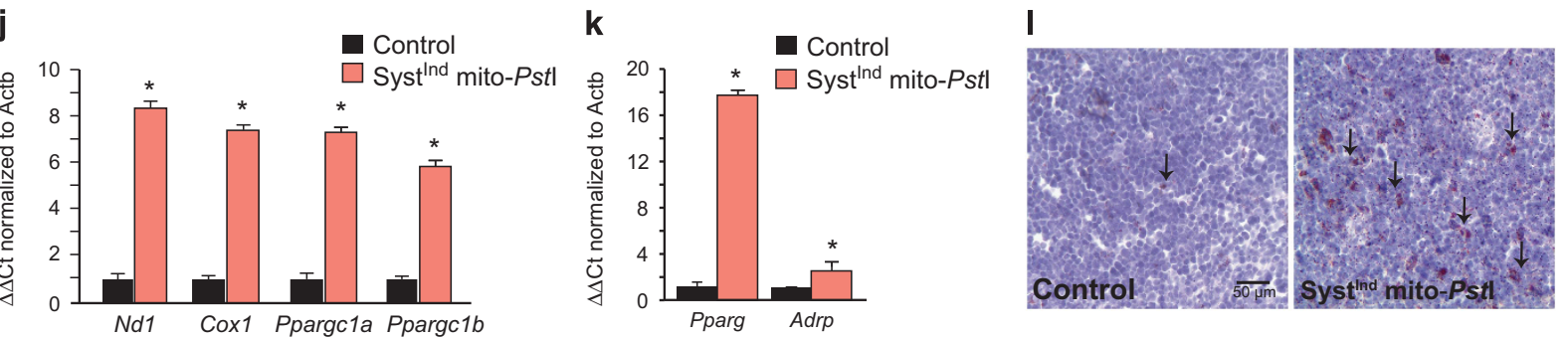

Figure 5 mtDNA damage in Systemic ${ }^{\text {Ind }}$ mito-Pstt mice causes a block in thymic progenitor progression leading to loss of thymocytes. (a) Picture of 12-month-old Systemic ${ }^{\text {Ind }}$ mito-Pstl mice compared to controls showing the complete absence of the thymus in transgenic animals. (b) Thymocyte counts from control and induced Systemic ${ }^{\text {Ind }}$ mito-Pstt mice at 3 months of age ( $n=3-4 /$ group). (c-d) FACS analysis and quantification of CD4+ and CD8+ surface antigens on thymocyte population from control and Systemic ${ }^{\text {Ind }}$ mito-Pstl mice (DN: double negative, DP: double positive). (e-f) FACS analysis and quantification of CD44+ and CD25+ surface antigens on the double negative thymocyte population from control and Systemic ${ }^{\text {Ind }}$ mito-Pstl mice at 3 months of age. (g) Schematic drawing of thymocyte stages of maturation with relative surface antigens expression. (h) Representative immunohistochemical images of cleaved caspase-3 staining (green) with DAPI nuclear counter stain (blue) in the thymus. M, medulla; $C$, cortex and quantification of caspase-3 positive cells in cortex and medulla of control and Systemic ${ }^{\text {Ind }}$ mito-Pstl mice. (i) $\beta$ - galactosidase activity in frozen thymic sections of control and Systemic ${ }^{\text {Ind }}$ mito-Pstl mice ( $n=2 /$ group). (j-I) Accelerated thymus senescence and adipose tissue differentiation in response to mtDNA damage in 3-month-old animals: (j) Fold expression of mtDNA-encoded transcripts (ND1 and COX1) and PGC-1 $\alpha / \beta$ mRNA levels in Systemic ${ }^{\text {Ind }}$ mito-Pst thymus compared to control mice ( $n=5 /$ group). (k) Fold expression of mRNA transcripts PPAR $\gamma$ and ADRP in Systemic ${ }^{\text {Ind }}$ mito-Pstt thymus compared to control mice ( $n=5 /$ group). (I) Oil Red O staining (red) with a hematoxylin counter stain (blue) of control and induced Systemic ${ }^{\text {Ind }}$ mito-Pstt thymus sections at $\times 20$ magnification ( $n=3 /$ group). Values are presented as mean $\pm S$.E.M. ${ }^{\star} P<0.05,{ }^{* *} P<0.01$, $\left.{ }^{* * *} P<0.001\right)$

of satellite cells (the stem cells in skeletal muscle). ${ }^{48}$ These observations suggested that the generation of mtDNA deletions themselves, which accumulate at low levels, did not drive the perceived premature aging-like phenotypes. Moreover, the majority of the aging-like phenotypes described affected tissues with proliferative potential (skin, bone marrow, testis) and progenitor cells. Similarly, we could not detect a marked depletion of mtDNA in the affected tissues coinciding with the appearance of the aging-like phenotypes, suggesting that these phenotypes are due to an earlier signaling event, not necessarily mtDNA depletion (or deletion) induced energy defects. 
a

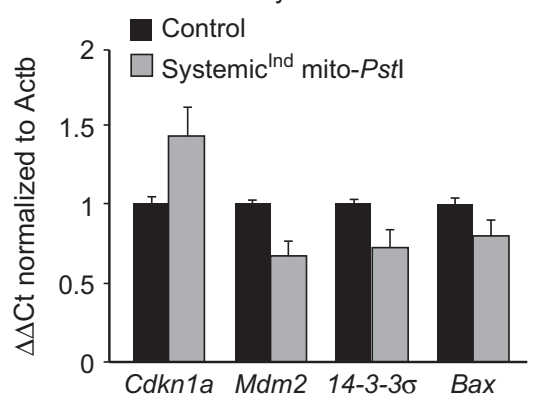

C

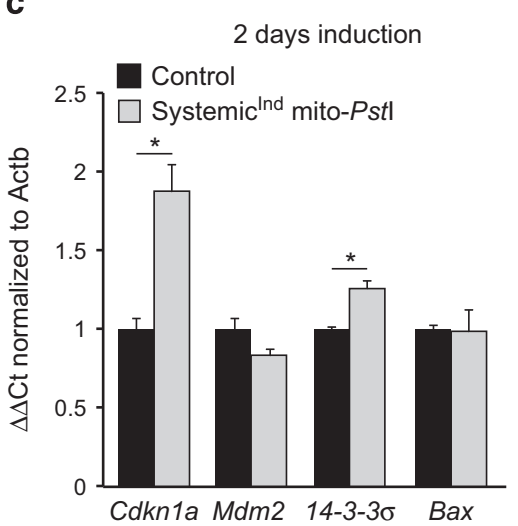

e

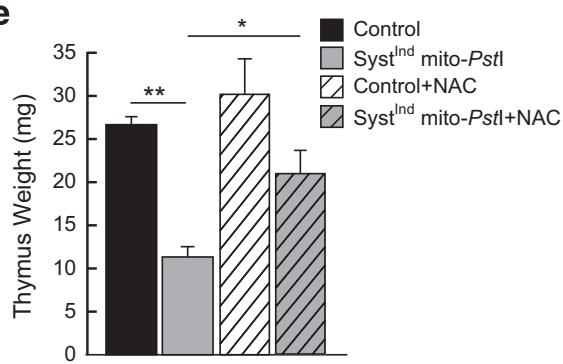

b

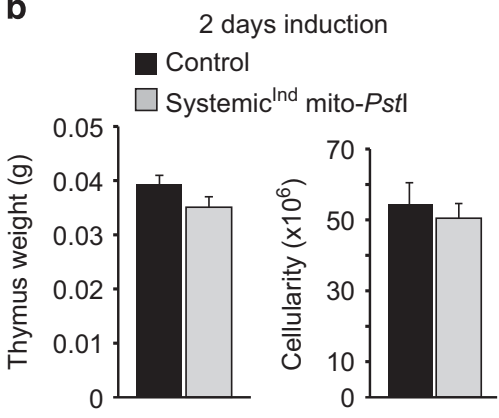

d

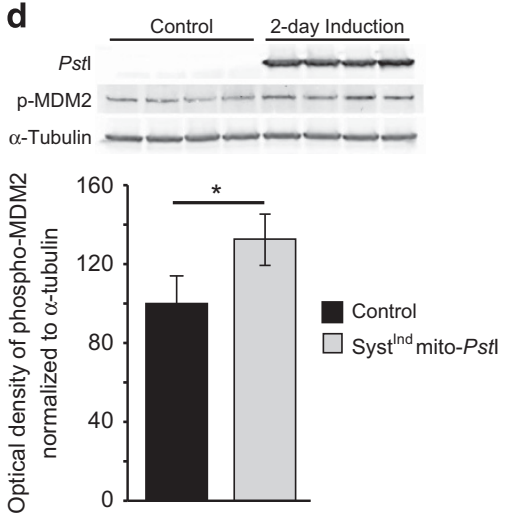

f

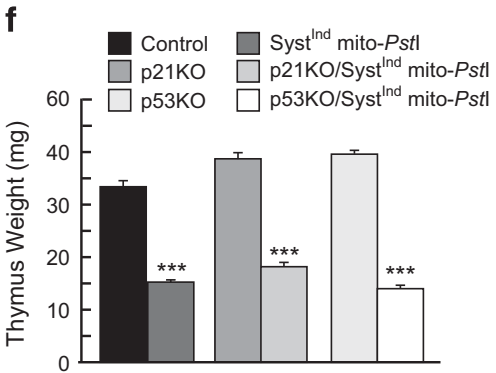

Figure 6 p53 transcriptional response is triggered by mtDNA damage in Systemic ${ }^{\text {Ind }}$ mito- $P$ stt thymus tissue but is not responsible for the aging phenotype. (a) Real-time RTPCR of p21 (Cdkn1a), 14-3-3 $\sigma$, Mdm2, and Bax transcripts in the thymus of 2-day and 5-day induced control or Systemic ${ }^{\text {Ind }}$ mito-Pstl mice ( $n=4-5 /$ group). (b) Thymus weight and cellularity after 2 days of induction of Systemic ${ }^{\text {Ind }}$ mito-Pstt mice. (d) Quantification of phosphorylated MDM2 protein levels in the thymus of 3-month-old Systemic ${ }^{\text {Ind }}$ mito-Pstl mice induced for 2 days over controls ( $n=4 /$ group). Values are presented as mean \pm S.E.M. $\left({ }^{*} P<0.05,{ }^{* \star} P<0.01,{ }^{* \star *} P<0.001\right)$. (e) Weight of thymus in control and Systemic ${ }^{\text {Ind }}$ mito-Pstl mice pre-treated with NAC (dashed columns): NAC alleviated the shrinkage of thymus in Systemic ${ }^{\text {Ind }}$ mito-Pstl mice. (f) Thymus weight in 3-month-old control, p21KO, p53KO, Systemic ${ }^{\text {Ind }}$ mito-Pstl, p21KO/Systemic ${ }^{\text {Ind }}$ mito-Pstl, p53KO/Systemic ${ }^{\text {Ind }}$ mito-Pstl: knocking out of p21 and p53 did not reverse or alleviate the shrinkage of the thymus in Systemic ${ }^{\text {Ind }}$ mito-Pstl mice

These results support the previous suggestion that progenitor cell pools are highly vulnerable to mitochondrialrelated ROS alterations. ${ }^{38,48}$ Ahlqvist et al. showed that the progeroid phenotype of the mutator mouse was blunted by NAC, which protected stem and progenitor cells. We also found that muscle satellite cells were decreased in the Systemic ${ }^{\text {Ind }}$ mito-Pstl mouse. ${ }^{48}$ Therefore, there is a growing body of evidence that mtDNA damage has a severe phenotypic effect in cells with high proliferative potential.

ROS effect: direct or indirect?. The ROS-associated p53 signaling observed appeared soon after mtDNA DSBs. We could not determine the source of this early ROS, although OXPHOS impairment would be the obvious candidate. It is difficult to explain the ROS generation without an OXPHOS dysfunction, but one could speculate that there are unidentified factors that recognize mtDNA DSBs and modify OXPHOS enzymes, leading to fast ROS production after mtDNA DSBs. Therefore, although we have found that ROS were the mediator of p53 signaling after mtDNA damage (in vitro), and that they were involved in the thymus shrinkage in Systemic ${ }^{\text {Ind }}$ mito-Pstl, the exact mechanism by which ROS is generated or signals is still unclear. Previous reports showed that ROS have the ability to activate p53 and inhibit ATM (upstream repressor of p53), without the presence of nuclear DNA damage. ${ }^{49}$ Even though we did not detect nuclear DNA damage in our in vitro system, it is still possible that the ROS from mtDNA insults could diffuse to the nucleus 
and damage nuclear DNA in the Systemic ${ }^{\text {Ind }}$ mito-Pst mice. ${ }^{50,51}$ In any case, the phenotypic alterations do not appear to depend on the p53 pathway (even if it is activated in the process), and may directly induce death of progenitor cells by a different mechanism. ROS signaling appears to be both activating the p53 pathway and another undefined pathway that leads to the p53-independent aging-like phenotypes observed. ${ }^{52}$

Other than ROS, different pathways are likely to be involved in the aging phenotype caused by mtDNA damage. One possibility is the activation of mtUPR system. Even though we did not explore this pathway in detail, we did not detect significant changes in the levels of mitochondrial chaperones (Hsp60, Hsp70) or of UPR markers (CHOP, BiP) in our in vitro mito-Scal system and in thymus of 2- and 5-day induced Systemic $^{\text {Ind }}$ mito-Pstl mice (Supplementary Figures S1F-H). Previously, ${ }^{48}$ we analyzed skeletal muscle after mito-Pst induction, and we detected a decrease in chaperones Hsp60, Hsp70, mtHsp70 and ER stress markers BiP/Grp78, CHOP, in 6-month-old systemic mito-Pstl mice muscles respect to controls. Although we cannot exclude that other components of this pathway may be activated, in different tissues and at different time points, we do not have evidence supporting this model.

\section{Conclusions}

MtDNA DSBs triggered differentiation, apoptosis, senescence and arrest of proliferative tissues: some stem and progenitor cells were markedly affected by this mechanism, possibly explaining the premature aging-like phenotypes observed in the Systemic ${ }^{\text {Ind }}$ mito-Pstl mouse. The aging phenotype, particularly in the thymus, was reverted by antioxidants, but not by knocking out p21 or p53, suggesting the involvement of other pathways associated with ROS signaling. This study demonstrates that mtDNA DSBs can have a major impact in tissue aging in a mechanism involving ROS, which can utilize, but does not require, p53 nor p21.

\section{Materials and Methods}

Ethics statement. The work with mouse models was approved by the University of Miami Institutional Animal Care and Use Committee (IACUC). As required by the IACUC, all efforts were done to minimize suffering of the animals.

Animals. The generation of mito- $P$ stl transgenic mice was previously described. ${ }^{28}$ Mito-Pst male and female animals were pure C57BL/6J mito-Pst (F10). Animals were crossed with Rosa26-rtTA animal of the same background (Jackson Laboratories, Bar Harbor, ME, USA). ${ }^{27}$ Systemic ${ }^{\text {Ind }}$ mito-Pstl mice were bred with p21KO and p53KO mice of the same background (Jackson Laboratories). ${ }^{39,40}$

All mice procedures were performed according to a protocol approved by the University of Miamil ACUC. When mito-PStl was induced at 3 months of age, we supplied both control and Systemic ${ }^{\text {Ind }}$ mito-Pstl animals with $10 \mathrm{~g} / \mathrm{kg}$ doxycycline diet (Bioserv, Flemington, NJ, USA) for 5 days and afterwards replaced with standard rodent diet.

For the NAC treatment, mice were given free access to drinking water starting 2 days before the DOX induction and during the 5 days of induction. Water was supplemented with $20 \mathrm{mM} \mathrm{NAC}$, which was calculated to yield an average dose of $2 \mathrm{~g}$ $\mathrm{NAC} / \mathrm{kg}$ body weight/day.

All experiments and comparisons described were performed on age- and gendermatched animals. Analyses were performed on both males and females separately and, unless noted, there were no significant changes between the genders.
Cell culture. The generation and maintenance of gorilla $\rho+$ and $\rho^{\circ}$ cell lines, and mito-Scal cell line were previously described. ${ }^{53}$ Mito-Scal cell line were treated with $10 \mu \mathrm{M}$ Etoposide (Sigma, St. Louis, MO, USA) for $24 \mathrm{~h}$. Mito-Pstt transfection was performed using GenJet In Vitro DNA Transfection Reagent according to the manufacturer's protocol and proteins were extracted after $24 \mathrm{~h}$ of transfection. NAC was added fresh and adjusted the $\mathrm{pH}$ to 7.4 .

Growth curves. Cells were counted and re-plated in 24-well plates at $5 \times 10^{\wedge} 3 \sim 10 \wedge 4$ cells/well. For growth curve cells were treated with $10 \mu \mathrm{M}$ mifepristone for $30 \mathrm{~min}$ or left untreated. Cells were counted in triplicate every $24 \mathrm{~h}$ using a TC10 Cell Counter (BioRad, Hercules, CA, USA) for up to $72 \mathrm{~h}$.

Western blotting. Western blotting procedures were previously described. ${ }^{54}$ Membranes were blocked in 1:1 Odyssey blocking solution (LI-COR Biosciences, Lincoln, NE, USA) or $5 \%$ milk for $1 \mathrm{~h}$ at room temperature.

Primary antibody was incubated overnight at $4{ }^{\circ} \mathrm{C}$. The following primary antibodies were used: HA 1: 1000 (Roche, Indianapolis, IN, USA), $\alpha$-tubulin and $\beta$ actin 1: 5000 (Sigma), Pst 1:100055), p21 1:1000 (BD Biosciences, San Jose, CA, USA), NDUFB8 1:1000 (Abcam, Cambridge, MA, USA), SOD-2 1:2000 (Millipore, Billerica, MA, USA). From Cell Signaling (Boston, MA, USA): phosphorylated MDM2 1:1000, p38 MAPK 1:2000, phospho-p38 MAPK 1:1000, JNK1 1:1000, phosphoSAPK/JNK 1:1000, CHOP 1:500, Hsp60 1:1000, Hsp70 1:1000, Grp78/BiP 1:1000.

Secondary antibodies used were either infrared conjugated antibodies anti-rabbit700/anti-mouse-800/anti-rat-800 (Rockland, Limerick, PA, USA) or HRP conjugated (Cell Signaling) at 1:3000-1:5000 concentrations. Secondary antibodies were incubated for $1 \mathrm{~h}$ at room temperature. Blots with infrared secondary antibodies were visualized with Odyssey Infrared Imaging System (LI-COR Biosciences). Blots with HRP secondary antibodies were visualized with Pico or Femto Supersignal West Chemiluminescent substrates (ThermoScientific, Waltham, MA, USA). Optical density measurements were taken by default software supplied by LI-COR or quantified using ImageJ.

RNA isolation and reverse transcriptase PCR. Dissected tissues or cells were submerged in TRIzol or TRI reagent (Sigma/Invitrogen, Waltham, MA, USA), and RNA was extracted. The iScript CDNA synthesis kit was used for reverse transcription reaction according to the manufacturer's protocol (BioRad).

Maxima SYBR Green/ROX qPCR master mix (Fermentas, Waltham, MA, USA) was used according to the manufacturer's directions to perform real-time PCR. Primers and sequences used for the CDNA quantification are available under request.

ND1 and $\beta$-actin (internal genomic DNA control) were used for the quantification of mtDNA copy number in total DNA. 8378F, $12821 \mathrm{~B}$ (small deletion $~ 3.8 \mathrm{~Kb}$ ); 5549F, $15501 \mathrm{~B}$ (large deletion $\sim 10.6 \mathrm{~Kb}$ ) were used for the quantification of the small and large deletion in total DNA. ${ }^{28}$ Comparative $\mathrm{Ct}$ method was used to determine the relative abundance of mtDNA or genes of interest. ${ }^{56}$

Thymocyte isolation and characterization. Single cell suspension of thymocytes was prepared and cell surface staining was performed as previously described. ${ }^{57}$ Conjugated antibodies PE-Cy7-CD4 and PE-CD25 were purchased from BD Biosciences (Franklin Lakes, NJ, USA). FITC-CD44 and Alexa700-CD8 were prepared in T.R. Malek's laboratory (Miami, FL, USA). FACS analyses were performed on an LSR-Fortessa-HTS flow cytometer with FACS Diva software (Becton Dickenson, Franklin Lakes, NJ, USA). 500000 events were obtained per sample.

Immunocytochemistry/immunohistochemistry. Cells were grown on top of glass coverslips. Cells were washed with PBS and fixed with $4 \%$ paraformaldehyde at room temperature. Cells were then treated with $0.1 \mathrm{M}$ glycine and permeabilized with $0.1 \%$ triton $X-100$. Cells were blocked in PBS containing $2 \%$ BSA and $2 \%$ normal goat serum. Primary antibody anti-HA $1: 100$ (Roche), H2AX $1: 500$ (Millipore) were incubated overnight at $4{ }^{\circ} \mathrm{C}$. AlexaFluor 488 or 594 goat antimouse/anti-rabbit/anti-rat (Invitrogen) antibodies were incubated for $45 \mathrm{~min}$ at room temperature. In experiments where mitochondria were visualized, MitoTracker Red ( $200 \mathrm{nM}$, Invitrogen) was added to the cells prior to fixation. Coverslips were mounted with Vectashield fluorescent mounting media with or without DAPI (Vector Laboratories, Burlingame, CA, USA). Images were taken with either an LSM510 or LSM710 confocal microscope (Zeiss, Thornwood, NY, USA). 
TUNEL staining. Cells were treated as indicated and TUNEL assay was performed according to the manufacturer's instructions (Roche). Images were taken with an LSM710 confocal microscope (Zeiss).

$\boldsymbol{\beta}$-Galactosidase staining. Thymus sections were processed as mentioned previously and cut at $10 \mu \mathrm{m}$. $\beta$-galactosidase staining was performed according to the manufacturer's instructions (Cell Signaling). Images were taken with an Olympus BX50.

Histology. After perfusing the mice with PBS, organs were extracted and submerged in formalin O.N. We analyzed intestine, testis and bone marrow. Bones were then treated in decalcifying solution (Sigma) for $8 \mathrm{~h}$ and then submerged again in formalin. Tissues were then embedded in paraffin and cut with a microtome. Twenty-micrometer sections were subjected to H\&E staining (Sigma). Images were taken with an Olympus BX50.

DEXA scanning. DEXA scans were performed using a Lunar PIXImus DEXA scan according to the manufacturer's instructions. Default software was used to quantify total/lean/fat mass, and bone mineral density.

Amplex Red assay. Hydrogen peroxide concentrations were measured by Amplex Red Peroxide/Peroxidase activity (Invitrogen) according to the manufacturer's directions. Fluorescence was read at excitation 570/emission 585 using a Synergy H1 hybrid plate reader (BioTek, Winooski, VT, USA). Fluorescence intensity was also normalized by cell number after the ending of the experiment.

Blood analysis and histological examination. Animals were anesthetized with a DVR-recommended ketamine cocktail, and blood was drawn from the right ventricle. Blood was placed in a plasma separation tube with heparin additives for analysis at the University of Miami Comparative Pathology Laboratory.

Oil Red $\mathrm{O}$ staining. Thymus was removed from animals and fixed overnight in $4 \%$ PFA. They were subsequently placed in a sucrose gradient $(10-30 \%)$ and frozen in Tissue Tek O.C.T (Sakura, Torrance, CA, USA) solution. Ten-micrometer sections were cut and stained $10 \mathrm{~min}$ in Oil Red $\mathrm{O}$ solution prepared according to the manufacturer's directions (EMS, Hatfield, PA, USA). Sections were counterstained for 2 min with Harris' hematoxylin stain (Sigma). Slides were rinsed and mounted in permanent aqueous mounting media (Covance, Hollywood, FL, USA). Images were taken on an Olympus BX50.

Statistics. Two-tailed, unpaired Student $t$-test was used to determine the statistical significance between the different groups. If more than two groups were analyzed, significance of the differences was evaluated by one-way ANOVA followed by Bonferroni post-test. Data are expressed as mean \pm S.E.M., or S.D. and the numbers of observations/animals used in each experimental series were included in the figure legends. ${ }^{\star} P<0.05,{ }^{* \star} P<0.01,{ }^{* \star} P<0.001$.

\section{Conflict of Interest}

The authors declare no conflict of interest.

Acknowledgements. We would like to thank Dr. Wayne E. Balkan from the University of Miami for the access to and the use of the DEXA scan; Dr. Norman $\mathrm{H}$. Altman VMD for consultation on thymus pathology, Dr. Ying Wang, and Dr. Ge Tao from the University of Miami for antibodies and technical assistance. We also thank the University of Miami Comparative Pathology Laboratory for the blood work analysis. This work was supported primarily by the US National Institutes of Health Grant 1R01AG036871. The following grants also helped support this work: $\mathrm{NIH}$ 1R01NS079965, 5R01EY010804; the Muscular Dystrophy Association and the United Mitochondrial Disease Foundation. We acknowledge support from the NEI center grant P30-EY014801 from the National Institutes of Health $(\mathrm{NIH})$.

1. Johnson FB, Sinclair DA, Guarente L. Molecular biology of aging. Cell 1999; 96: 291-302.

2. Sedelnikova OA, Horikawa I, Zimonjic DB, Popescu NC, Bonner WM, Barrett JC. Senescing human cells and ageing mice accumulate DNA lesions with unrepairable doublestrand breaks. Nat Cell Biol 2004; 6: 168-170.

3. Vogel H, Lim DS, Karsenty G, Finegold M, Hasty P. Deletion of Ku86 causes early onset of senescence in mice. Proc Natl Acad Sci USA 1999; 96: 10770-10775.
4. Murga M, Bunting S, Montana MF, Soria R, Mulero F, Canamero M et al. A mouse model of ATR-Seckel shows embryonic replicative stress and accelerated aging. Nat Genet 2009; 41: 891-898.

5. Inomata K, Aoto T, Binh NT, Okamoto N, Tanimura S, Wakayama T et al. Genotoxic stress abrogates renewal of melanocyte stem cells by triggering their differentiation. Cell 2009; 137: 1088-1099.

6. Ruzankina Y, Schoppy DW, Asare A, Clark CE, Vonderheide RH, Brown EJ. Tissue regenerative delays and synthetic lethality in adult mice after combined deletion of Atr and Trp53. Nat Genet 2009; 41: 1144-1149.

7. Ruzankina Y, Pinzon-Guzman C, Asare A, Ong T, Pontano L, Cotsarelis G et al. Deletion of the developmentally essential gene ATR in adult mice leads to age-related phenotypes and stem cell loss. Cell Stem Cell 2007; 1: 113-126.

8. Rodier F, Campisi J, Bhaumik D. Two faces of p53: aging and tumor suppression. Nucleic Acids Res 2007; 35: 7475-7484.

9. Renault VM, Thekkat PU, Hoang KL, White JL, Brady CA, Kenzelmann Broz D et al. The pro-longevity gene $\mathrm{FoxO} 3$ is a direct target of the p53 tumor suppressor. Oncogene 2011; 30: 3207-3221.

10. Gudkov AV, Gurova KV, Komarova EA. Inflammation and p53: a tale of two stresses. Genes Cancer 2011; 2: 503-516.

11. Feng $Z$, Lin M, Wu R. The regulation of aging and longevity: a new and complex role of $p 53$. Genes Cancer 2011; 2: 443-452.

12. Balaban RS, Nemoto S, Finkel T. Mitochondria, oxidants, and aging. Cell 2005; 120 : 483-495.

13. Schumacher B, van der Pluijm I, Moorhouse MJ, Kosteas T, Robinson AR, Suh $Y$ et al. Delayed and accelerated aging share common longevity assurance mechanisms. PLoS Genet 2008; 4: e1000161.

14. McCarroll SA, Murphy CT, Zou S, Pletcher SD, Chin CS, Jan YN et al. Comparing genomic expression patterns across species identifies shared transcriptional profile in aging. Nat Genet 2004; 36: 197-204.

15. Zahn JM, Sonu R, Vogel H, Crane E, Mazan-Mamczarz K, Rabkin R et al. Transcriptional profiling of aging in human muscle reveals a common aging signature. PLOS Genet 2006; 2 : e115.

16. Anderson S, Bankier AT, Barrell BG, de Bruijn MH, Coulson AR, Drouin J et al. Sequence and organization of the human mitochondrial genome. Nature 1981; 290: 457-465.

17. Harman D. Aging: a theory based on free radical and radiation chemistry. J gerontol 1956 ; 11: $298-300$

18. Wallace DC. Mitochondrial DNA mutations in disease and aging. Environ mol mutagen 2010; 51: $440-450$.

19. Vermulst M, Bielas JH, Kujoth GC, Ladiges WC, Rabinovitch PS, Prolla TA et al. Mitochondrial point mutations do not limit the natural lifespan of mice. Nat Genet 2007; 39: 540-543.

20. Vermulst M, Wanagat J, Kujoth GC, Bielas JH, Rabinovitch PS, Prolla TA et al. DNA deletions and clonal mutations drive premature aging in mitochondrial mutator mice. Nat Genet 2008; 40: 392-394.

21. Schaar CE, Dues DJ, Spielbauer KK, Machiela E, Cooper JF, Senchuk M et al. Mitochondrial and cytoplasmic ROS have opposing effects on lifespan. PLOS genet2015; 11 : e1004972.

22. Van Raamsdonk JM, Hekimi S. Deletion of the mitochondrial superoxide dismutase sod-2 extends lifespan in Caenorhabditis elegans. PLoS genet 2009; 5: e1000361.

23. Bacman SR, Williams SL, Moraes CT. Intra- and inter-molecular recombination of mitochondrial DNA after in vivo induction of multiple double-strand breaks. Nucleic acids res 2009; 37: 4218-4226.

24. Zeke A, Misheva M, Remenyi A, Bogoyevitch MA. JNK signaling: regulation and functions based on complex protein-protein partnerships. Microbiol mol biol rev 2016; 80: 793-835.

25. Munshi A, Ramesh R. Mitogen-activated protein kinases and their role in radiation response. Genes cancer 2013; 4: 401-408.

26. Lee SB, Bae IH, Bae YS, Um HD. Link between mitochondria and NADPH oxidase 1 isozyme for the sustained production of reactive oxygen species and cell death. J Biol Chem 2006; 281: 36228-36235.

27. Belteki G, Haigh J, Kabacs N, Haigh K, Sison K, Costantini F et al. Conditional and inducible transgene expression in mice through the combinatorial use of Cre-mediated recombination and tetracycline induction. Nucleic Acids Res 2005; 33: e51.

28. Fukui $\mathrm{H}$, Moraes CT. Mechanisms of formation and accumulation of mitochondrial DNA deletions in aging neurons. Hum mol genet 2009; 18: 1028-1036.

29. Awong G, Zuniga-Pflucker JC. Thymus-bound: the many features of T cell progenitors. Front Biosci (Schol Ed) 2011; 3: 961-969.

30. Marinova TT. Epithelial framework reorganization during human thymus involution. Gerontology 2005; 51: 14-18.

31. Puigserver P, Wu ZD, Park CW, Graves R, Wright M, Spiegelman BM. A cold-inducible coactivator of nuclear receptors linked to adaptive thermogenesis. Cell 1998; 92: 829-839.

32. Rosen ED, Sarraf P, Troy AE, Bradwin G, Moore K, Milstone DS et al. PPAR gamma is required for the differentiation of adipose tissue in vivo and in vitro. Mol Cell 1999; 4: $611-617$.

33. Brun RP, Tontonoz P, Forman BM, Ellis R, Chen J, Evans RM et al. Differential activation of adipogenesis by multiple PPAR isoforms. Gene Dev 1996; 10: 974-984.

34. Lozano G, Levine AJ. Tissue-specific expression of P53 in transgenic mice is regulated by intron sequences. Mol Carcinogen 1991; 4: 3-9. 
35. Tyner SD, Venkatachalam S, Choi J, Jones S, Ghebranious N, Igelmann H et al. p53 mutant mice that display early ageing-associated phenotypes. Nature 2002; 415: 45-53.

36. Liu DP, Ou LD, Clemenson GD, Chao C, Lutske ME, Zambetti GP et al. Puma is required for p53-induced depletion of adult stem cells. Nat Cell Biol 2010; 12: 993-998.

37. Lavin MF, Gueven N. The complexity of p53 stabilization and activation. Cell Death Differ 2006; 13: 941-950.

38. Ahlqvist KJ, Hamalainen RH, Yatsuga S, Uutela M, Terzioglu M, Gotz A et al. Somatic progenitor cell vulnerability to mitochondrial dna mutagenesis underlies progeroid phenotypes in polg mutator mice. Cell Metab 2012; 15: 100-109.

39. Deng C, Zhang P, Harper JW, Elledge SJ, Leder P. Mice lacking p21CIP1/WAF1 undergo normal development, but are defective in G1 checkpoint control. Cell 1995; 82 675-684.

40. Jacks T, Remington L, Williams BO, Schmitt EM, Halachmi S, Bronson RT et al. Tumor spectrum analysis in p53-mutant mice. Curr biol 1994; 4: 1-7.

41. Coffey G, Campbell C. An alternate form of Ku80 is required for DNA end-binding activity in mammalian mitochondria. Nucleic Acids Res 2000; 28: 3793-3800.

42. Lakshmipathy U, Campbell C. Mitochondrial DNA ligase III function is independent of xrcc1. Nucleic Acids Res 2000; 28: 3880-3886.

43. Lakshmipathy U, Campbell C. The human DNA ligase III gene encodes nuclear and mitochondrial proteins. Mol Cell Biol 1999; 19: 3869-3876.

44. Eaton JS, Lin ZP, Sartorelli AC, Bonawitz ND, Shadel GS. Ataxia-telangiectasia mutated kinase regulates ribonucleotide reductase and mitochondrial homeostasis. J Clin Invest 2007; 117: 2723-2734

45. Valentin-Vega YA, MacLean KH, Tait-Mulder J, Milasta S, Steeves M, Dorsey FC et al. Mitochondrial dysfunction in ataxia-telangiectasia. Blood 2012; 119: 1490-1500.

46. Sage JM, Gildemeister OS, Knight KL. Discovery of a novel function for human Rad51 maintenance of the mitochondrial genome. J Biol Chem 2010; 285: 18984-18990.
47. Bayona-Bafaluy MP, Blits B, Battersby BJ, Shoubridge EA, Moraes CT. Rapid directional shift of mitochondrial DNA heteroplasmy in animal tissues by a mitochondrially targeted restriction endonuclease. Proc Natl Acad Sci USA 2005; 102: 14392-14397.

48. Wang X, Pickrell AM, Rossi SG, Pinto M, Dillon LM, Hida A et al. Transient systemic mtDNA damage leads to muscle wasting by reducing the satellite cells pool. Hum mol genet 2013; 22: 3976-3986.

49. Guo Z, Kozlov S, Lavin MF, Person MD, Paull TT. ATM activation by oxidative stress. Science 2010; 330: 517-521.

50. Hartman P, Ponder R, Lo HH, Ishii N. Mitochondrial oxidative stress can lead to nuclear hypermutability. Mech Ageing Dev 2004; 125: 417-420.

51. Lauritzen KH, Cheng C, Wiksen H, Bergersen LH, Klungland A. Mitochondrial DNA toxicity compromises mitochondrial dynamics and induces hippocampal antioxidant defenses. DNA Repair 2011; 10: 639-653.

52. Pohjoismaki JL, Williams SL, Boettger T, Goffart S, Kim J, Suomalainen A et al. Overexpression of Twinkle-helicase protects cardiomyocytes from genotoxic stress caused by reactive oxygen species. Proc Natl Acad Sci USA 2013; 110: 19408-19413.

53. Bacman SR, Williams SL, Moraes CT. Intra- and inter-molecular recombination of mitochondrial DNA after in vivo induction of multiple double-strand breaks. Nucleic Acids Res 2009; 37: 4218-4226.

54. Pickrell AM, Fukui $H$, Wang $X$, Pinto $M$, Moraes $C T$. The striatum is highly susceptible to mitochondrial oxidative phosphorylation dysfunctions. J neurosci 2011; 31: 9895-9904.

55. Srivastava S, Moraes CT. Manipulating mitochondrial DNA heteroplasmy by a mitochondrially targeted restriction endonuclease. Hum mol genet 2001; 10: 3093-3099.

56. Schmittgen TD, Livak KJ. Analyzing real-time PCR data by the comparative C-T method. Nat Protoc 2008; 3: 1101-1108.

57. Bayer AL, Yu AX, Malek TR. Function of the IL-2R for thymic and peripheral CD4(+)CD25(+) Foxp3(+) T regulatory cells. J Immunol 2007; 178: 4062-4071.

Supplementary Information accompanies this paper on Cell Death and Differentiation website (http://www.nature.com/cdd) 